\title{
The effect of sodium phosphate enema in patients ingested foreign bodies: a prospective, randomized trial
}

\author{
_- Sodium phosphate enema in patients ingested foreign bodies \\ Zeynep Ozkan $^{1}$, Metin Kement ${ }^{1 *}$, Mustafa Oncel ${ }^{1,2}$, Levent Kaptanoglu ${ }^{1}$, Cem Gezen ${ }^{1}$ \\ ${ }^{1}$ Department of General Surgery, Kartal Education and Research Hospital, Istanbul, Turkey; drzeynepozkan@yahoo.com; \\ *Corresponding Author: mkement@yahoo.com; oncel@hotmail.com; leventkaptanoglu@yahoo.com; cemgezen@hotmail.com; \\ ${ }^{2}$ Gumushane Univercity College of Health Sciences, Gumushane, Turkey.
}

Received 14 August 2010; revised 20 August 2010; accepted 23 August 2010.

\section{ABSTRACT}

Background: Although foreign body ingestion is a common problem, poor knowledge for the treatment still remains a major obstacle, especially in preventing complications. Phosphate enemas were applied for chronic constipation and bowel cleansing for a long period of time. This study aims to evaluate the effect of phosphate enema on bowel movements in patients who suffer from foreign body ingestion. Methods: Forty consecutive patients admitted to our emergency department for foreign body ingestion were randomized into two groups. Cases in Group SP received daily one dose of sodium phosphate enemas, while Group $C$ did not. Patients in both groups were followed up with daily imaging and physical examinations. Demographics, number of $X$-rays, radiation dose, and period of the foreign body passage were recorded and compared between two groups. Also, data of patients who ingested pins (Group P) were compared with of those who ingested other materials (Group 0). Results: There was no statistical difference between groups (Group SP vs. Group C) comparing demographics, number of $X$-rays, total amount of radiation doses $(p>0.05$ for all comparisons). The period of foreign body passage was shorter in Group SP $(3.4 \pm 1.6$ days vs. $6.2 \pm 3.8$ days; $p=0.005$ ). Also, patients who ingested pins (Group P) were compared to patients who ingested other materials (Group 0 ). Pins were ingested by $23(65 \%)$ patients, these were older $(16.6 \pm 9.4$ years vs. $4.8 \pm 3.5$ years; $p$ $=0.001)$ and generally females $(78.3 \%$ vs. $29.4 \%$; $p=0.006)$. Contrary to the other materials, pins were less frequently confirmed in stool of patients $(p=0.02)$. Conclusion: Sodium phosphate enema use may hasten the passage of the foreign bodies. Ingestion of pins is the more common especially in turbaned young females. The evacuation of pins is less recognized with direct examination of the stool, so daily imaging may be necessary for this group of patients.

Keywords: Sodium Phosphate Enema; Foreign Body Ingestion

\section{INTRODUCTION}

The ingestion of foreign bodies is a common problem among patients admitted to emergency units. Although ingestion of a foreign body is generally associated with little or no morbidity, and thus often underestimated; serious complications, such as perforation, bleeding or obstruction are also reported [1-3]. Most cases are preschool age children; however the aspiration or ingestion of turban pins has been encountered among adolescent girls in Islamic countries, where turban is worn as a kind of head cover for religious intentions [3-6].

The heterogeneity in the type of ingested foreign body has limited the development of an algorithm for management. If the foreign body passes the pylorus, followup is indicated to rule out complications until the material is evacuated. However, the details of protocols, such as whether or not daily radiographies are necessitated, are still debated [7]. Since the majority of materials are radio-opaque, a frontal radiograph may be helpful for the diagnosis. Endoscopic removal is generally possible if the material is located in the esophagus. Some have suggested the extraction of the material with a Foley catheter, since it is a cost-effective approach, or the use of glucagon and water as an effective treatment for the esophageal foreign bodies [8,9]. However, the best of our knowledge, there have been no prospective studies 
for the evaluation of any treatment modality when the ingested foreign material is located beyond the pylorus, where endoscopic removal is almost impossible.

Sodium phosphate enema is useful as a laxative in the relief of occasional constipation and as part of bowel cleansing regimen in preparing the colon for surgery, $\mathrm{x}$-ray or endoscopic examination. It has been long used for the treatment of chronic constipation [10]. Although the effect of sodium phosphate enema on bowel movements has never been evaluated, it generally induces complete emptying of the left colon. Its use is safe even in children, and side-effects have been reported in only a small number of cases. This study aims to evaluate the effect of sodium phosphate enema in patients who ingested foreign material.

\section{MATERIALS AND METHODS}

This study was approved by the Hospital Education Planning Committee and conducted in Kartal Education and Research Hospital between March 2003 and August 2005. Written informed consent was obtained from all patients or from their parents if the patient was under 18 year-old.

The consecutive patients who admitted to our emergency department with foreign body ingestion between March 2003 and August 2005 were included in this study. Patients younger than 6 month-old or pregnant were excluded because sodium phosphate enema or radiological examinations might be unsafe. Patients were also excluded if an endoscopic procedure or surgery was necessitated due to esophageal material, extralumination of the foreign body or the presence of acute abdomen at admission.

All enrolled patients were randomized with a computerized algorithm into two groups. Patients in Group SP received a single dose of sodium phosphate enema (135 $\mathrm{ml}$ for adults and $3 \mathrm{ml} / \mathrm{kg}$ for children; B.T. Enema Sol, Yenisehir Drugs, Turkey) immediately after the admission to emergency unit and they were instructed to use a single daily dose of sodium phosphate enema. Group C was control group; the patients in this group did not receive any treatment and were only observed.

In this study, another analysis was made by comparing the patients who ingested pins (Group P) with of those who ingested other materials (Group O).

All patients were followed up with daily X-ray and physical examination until the evacuation was confirmed by the absence of the material in the X-rays or by direct visualization of the foreign body in the stool.

These were obtained prospectively and compared within the groups: Demographics, period between the ingestion and admission, characteristics of the materials, period of the foreign body passage, number of X-ray ex- aminations and exposed radiation dose. The data were compared within Group SP and Group C. Also, data of the patients who ingested pins (Group P) were compared with of those who ingested other materials (Group O).

Statistics: All statistical analyses were done with SPSS for Windows 10.0. The results were presented as percenttages, means, standard deviations and ranges. Student ttest and Pearson's Chi-square test were used for evaluation of numeric and continuing values and a $\mathrm{p}$ value less than 0.05 was considered significant.

\section{RESULTS}

Forty patients admitted to our emergency department due to foreign body ingestion were randomized into two groups. There were $23(57.5 \%)$ females, and the median age was 11 years old (1-39 years). Most patients were under 10 years old $(n=21 ; 52.5 \%)$ or teenager (age between 10 and 20 years old) $(n=15 ; 37.5 \%)$, and only 6 $(15.0 \%)$ patients were older than 20 years old. The mean period between the ingestion and hospital admission was $113.5 \pm 176$ (20-900) minutes. The most commonly ingested material was pin $(\mathrm{n}=23 ; 57.5 \%)$. The demographic data and the characteristics of the foreign materials were similar within the groups (Table 1). In study period, none of our patients developed any complications such as bowel obstruction or perforation.

The passage period of the material was more than 5 days in $1(5.0 \%)$ and $8(40.0 \%)$ in Groups SP and C, respectively, and significantly shorter in Group SP than in Group C $(\mathrm{p}=0.005)$ (Table 1). However, the number of X-ray examinations and the total amount of radiation given to the patients were similar in Groups SP and C (Table 1).

In a retrospective analysis, the patients who ingested pins (Group P; $n=23$ ) were compared to those ingested other materials (Group $\mathrm{O} ; \mathrm{n}=17$ ). Females were majority population in Group $\mathrm{P}(\mathrm{p}=0.006)$ and they were older than patients in Group O $(\mathrm{p}=0.001)$ (Table 2). The number of patients received sodium phosphate enema treatment, the passage period of the material, number of X-ray examinations and doses of radiation were similar within the groups. Foreign body could not be visualized in the stool in more patients in Group $\mathrm{P}$ than in those in Group $\mathrm{O}(\mathrm{p}=0.02)$.

\section{DISCUSSION}

Foreign body ingestion is quite common problem. Most of the foreign bodies that reach the gastrointestinal tract will pass spontaneously. However, $10 \%$ to $20 \%$ of them will require nonoperative intervention such as endoscopy, and approximately $1 \%$ of them will require surgery [11-13]. In most instances, the ingestion goes 
Table 1. Comparison of Group SP and Group C by demographics and clinical characteristics.

\begin{tabular}{|c|c|c|c|}
\hline & Group SP $(n=20)$ & Group C $(\mathrm{n}=20)$ & $\mathrm{p}$ \\
\hline \multicolumn{4}{|l|}{ Demographics } \\
\hline Age $[$ Mean $\pm \mathrm{SD}$ (range) in years $]$ & $11.1 \pm 9.4(1-33)$ & $12.0 \pm 8.5(1-39)$ & 0.85 \\
\hline Gender [Females (\%)] & $11(55)$ & $12(60)$ & 0.75 \\
\hline Admission Period [Median (range) in minutes] & $120.5 \pm 188.9(20-900)$ & $106.5 \pm 166.8(20-800)$ & 0.80 \\
\hline \multicolumn{4}{|l|}{ Type of The Material } \\
\hline Pin $(\%)$ & $10(50)$ & $13(65)$ & \multirow{8}{*}{0.74} \\
\hline Coin $(\%)$ & $4(20)$ & $4(20)$ & \\
\hline Screw $(\%)$ & $2(10)$ & $0(0)$ & \\
\hline Safety pin (\%) & $1(5)$ & $1(5)$ & \\
\hline Earring (\%) & $1(5)$ & $1(5)$ & \\
\hline Coil $(\%)$ & $1(5)$ & $0(0)$ & \\
\hline Peg $(\%)$ & $1(5)$ & $0(0)$ & \\
\hline Ring (\%) & $0(0)$ & $1(5)$ & \\
\hline Passage Period of The Material [Mean $\pm \mathrm{SD}$ (range) in days] & $2.7 \pm 1.3(1-6)$ & $5 \pm 3.9(1-18)$ & $0.016 *$ \\
\hline Number of X-ray Examinations [Mean $\pm \mathrm{SD}$ (range)] & $2.5 \pm 0.7(1-4)$ & $3.2 \pm 1.8(1-10)$ & 0.12 \\
\hline Radiation Dose [Mean \pm SD (range) in miliREM] & $775 \pm 217(310-1210)$ & $992 \pm 558(310-2100)$ & 0.12 \\
\hline
\end{tabular}

(*: Statistically significant. The data were given as means and ranges or percentages. The Student t-test and Pearson's Chi-square test were used for evaluation of numeric and continuing values and a $p$ value less than 0.05 was considered significant).

Table 2. Comparison of Group P and Group O by demographics and clinical characteristics.

\begin{tabular}{|c|c|c|c|}
\hline & Group $P(n=23)$ & Group O $(\mathrm{n}=17)$ & $\mathrm{p}$ \\
\hline \multicolumn{4}{|l|}{ Demographics } \\
\hline Age $[$ Mean $\pm \mathrm{SD}$ (range) in years & $16.6 \pm 9.4(1-39)$ & $4.8 \pm 3.5(1-14)$ & 0.001* \\
\hline Gender [Females (\%)] & $18(78.3)$ & $5(29.4)$ & 0.006* \\
\hline \multicolumn{4}{|l|}{ End of Follow-up } \\
\hline by the Direct Vision of the Material in the Stool (\%) & $7(34.4)$ & $12(70.8)$ & \multirow{2}{*}{$0.02 *$} \\
\hline by the Absence of the Material in the X-rays (\%) & $16(69.6)$ & $5(29.4)$ & \\
\hline Passage Period of The Material [Mean \pm SD (range) in days] & $3.7 \pm 3.8(1-18)$ & $4 \pm 2.4(1-10)$ & 0.73 \\
\hline Number of X-ray Examinations & $3.1 \pm 1.9(1-10)$ & $2.6 \pm 0.9(1-7)$ & 0.32 \\
\hline Radiation Dose [Mean (range) in miliREM] & $962 \pm 589(310-3100)$ & $806 \pm 279(310-2170)$ & 0.32 \\
\hline Number of patients received sodium phosphate enema treatment (\%) & $13(56.5)$ & $7(41.2)$ & 0.34 \\
\hline
\end{tabular}

(*: Statistically significant. The data were given as means and ranges or percentages. The Student t-test and Pearson's Chi-square test were used for evaluation of numeric and continuing values and a $\mathrm{p}$ value less).

unrecognized or unreported until the onset of symptoms. Children, the mentally impaired, or the psychiatric patients may present with refusal to eat, vomiting, choking, drooling, wheezing, bloodstained saliva, or respiratory distress. Erythema, tenderness, or crepitus in the neck may be present with oropharyngeal or esophageal perforation. The abdomen should be examined for evidence of small bowel perforation or peritonitis. These conditions will require surgical intervention. Ventilation, airway compromise and the risk of aspiration should also be assessed. 
Radiographs can mostly identify foreign bodies and free mediastinal or peritoneal air. The lateral projection confirms location in the esophagus and may reveal the presence of more than one coin. However, non-metallic objects are not readily seen. A contrast examination should not be performed routinely because of the risk of aspiration and coating of the foreign body and esophageal mucosa compromises subsequent endoscopy. If symptoms are not clear or specific, a cautious contrast study may be appropriate to clarify the presence of a foreign body or its location. Computerized tomography may be useful in some cases. Metal detectors can detect swallowed metallic objects and may be of use as a screening tool especially in pediatric patients [14]. Persistent symptoms related to the esophagus in cases of suspected foreign body ingestion should be pursued with endoscopy even after an apparently unrevealing radiographic evaluation.

Management of foreign body ingestion is influenced by many factors such as patient's age and clinical condition; size, shape, and classification of the ingested material; the anatomic location in which the object is lodged [15]. The physician should decide whether endoscopic or surgical intervention is necessary or not, what degree of urgency is called for, and by what means. In medication, smooth-muscle relaxation agents such as benzodiazepines, and nifedipine and those which improve peristaltic activity such as glucagon and E-Z Gas may be used. But, there is not convincing evidence in the literature that the use of such agents changes clinical outcomes [16].

This study is the first to evaluate the effect of sodium phosphate enema in patients who swallowed foreign materials. The results of the study indicate that the use of enema shortens the period between the ingestion and evacuation of the material, however, do not change the number of necessitated $\mathrm{x}$-rays, or the radiation dose administrated to the patients. In our opinion, this shortening effect of enema was due to emptying of the rectum. Rectal distension with feces produces an inhibitory effect on jejunal and ileal peristalsis. This effect was termed as recto-enteric reflex [17]. Rectal emptying by enema decreases rectal distension and in this way may inhibit recto-enteric reflex.

Contrary to the literature, pins were the most commonly ingested material in our study, because most of our patients were young Muslim girls who inadvertently swallowed turban pins. Also, the proportion of children was less than the western studies in our study because of high number of young girls [1-3]. Thus we, retrospectively, compared the characteristics between patients who ingested pins versus other materials. We have found that the pin ingestion mostly occurs in turbaned young girls. Thus, in our opinion, pin ingestion is a completely different entity according to demographic evaluation. This analysis revealed that direct visualization of the pins in the stool is difficult, so the defecation of material was inferred by the absence of pins on abdominal radiographs in almost two-thirds of the patients in this group. Although radiograph of stool would be an option to avoid radiation exposure to young girls, daily radiography is frequently necessary in patients who ingest pin.

\section{CONCLUSIONS}

Sodium phosphate enemas may be beneficial in patients who suffer from foreign body ingestion, since it is completely safe, easy-to-use, well-accepted by the patients, and it may hasten the pass over period of the materials.

As a subgroup of swallowed foreign materials, pin ingestion is more common among turbaned young girls in our country.

\section{REFERENCES}

[1] Paul, R.I., Christoffel, K.K., Binns, H.J., Jaffe, D.M. and the Pediatric Practice Research Group. (1993) Foreign body ingestion in children: Risk of complication varies with site of initial health care contact. Pediatrics, 91(1), 121-127.

[2] Hashmonai, M., Kaufman, T. and Schramek, A. (1978) Silent perforations of the stomach and duodenum by needles. Archives of Surgery, 113(12), 1406-1409.

[3] Cheng, W. and Tam, P.K.H. (1999) Foreign-body ingestion in children: Experience with 1,265 cases. Journal of Pediatric Surgery, 34(10), 1472-1476.

[4] Kaptanoglu, M., Dogan, K., Onen, A. and Kunt, N. (1999) Turban pin aspiration: A potential risk for young Islamic girls. International Journal of Pediatric Otorhinolaryngology, 48(2), 131-135.

[5] Shabb, B., Taha, A.M., Hamada, F. and Kanj, N. (1996) Straight pin aspiration in young women. Journal of Trauma, 40(5), 827-828.

[6] Ucan, E.S., Tahaoglu, K., Mogolkoc, N., Dereli, S., Basoz-demir, N., Basok, O., Turktas, H., Akkocoglu, A. and Ates, M. (1996) Turban pin aspiration syndrome: A new form of foreign body aspiration. Respiratory Medicine, 90(7), 427-428.

[7] Hodge III, D., Tecklenburg, F. and Fleisher, G. (1985) Coin ingestion: Does every child need a radiograph? $\mathrm{An}$ nals of Emergency Medicine, 14(5), 443-446.

[8] Kelley, J.E., Leech, M.H. and Carr, M.G. (1993) A safe and cost-effective protocol for the management of esophageal coins in children. Journal of Pediatric Surgery, 28(7), 898-900.

[9] Robbins, M.I. and Shortsleeve, M.J. (1994) Treatment of acute esophageal food impaction with glucagons, an effervescent agent, and water. American Journal of Roentgenology, 162, 325-328.

[10] Davies, C. (2004) The use of phosphate enemas in the treatment of constipation. Nursing Times, 100(18), 32-35.

[11] Webb, W.A. (1995) Management of foreign bodies of the upper gastrointestinal tract: Update. Gastrointest Endosc, 
41(1), 39-51.

[12] Nandi, P. and Ong, G.B. (1978) Foreign body in the esophagus: Review of 2394 cases. British Journal of Surgery, 65, 5-9.

[13] Vizcarrondo, F.J., Brady, P.G. and Nord, H.J. (1983) Foreign bodies of the upper gastrointestinal tract. Gastrointest Endosc, 29(3), 208-210.

[14] Doraiswamy, N.V., Baig H. and Hallam, L. (1999) Metal detector and swallowed metal foreign bodies in children. Journal of Accident \& Emergency, 16(2), 123-125.
[15] Ginsberg, G.G. (1995) Management of ingested foreign objects and food bolus impactions. Gastrointest Endosc, 41(3), 33-38.

[16] Al-Haddad, M., Ward, E.M., Scolapio, J.S., Ferguson, D.D. and Raimondo, M. (2006) Glucagon for the relief of esophageal food impaction does it really work. Digestive Diseases Sciences, 51(11), 1930-1933.

[17] Shafik, A. (2000) Effect of rectal distension on the small intestine with evidence of a recto-enteric reflex. Hepatogastroenterology, 47(34), 1030-1033. 